\title{
Numerical simulations of Jupiter Family Comets; physical and dynamical effects
}

\author{
Romina P. Di Sisto ${ }^{1}$, Julio A. Fernández ${ }^{2}$ and Adrián Brunini ${ }^{1}$ \\ ${ }^{1}$ Facultad de Ciencias Astronómicas y Geofísicas - UNLP, \\ IALP - CONICET, Paseo del Bosque $S / N$, La Plata. Argentina \\ email: romina@fcaglp.unlp.edu.ar, abrunini@fcaglp.unlp.edu.ar \\ ${ }^{2}$ Departamento de Astronomía, Facultad de Ciencias, Iguá 4225, 11400, Montevideo. Uruguay. \\ email: julio@fisica.edu.uy
}

\begin{abstract}
We present results from numerical simulations of a Jupiter family comet (JFC) population (orbital periods $P<20 \mathrm{yr}$ and Tisserand parameters in the range $2<T<3.1$ ) originated in the Scattered Disk and transferred to the Jupiter's zone through gravitational interactions with the Jovian planets. We shall call 'non-JFCs' those comets coming from the same source, but that do not fulfill the previous criteria (mainly because they have periods $P>20 \mathrm{yr}$ ). We have carried out series of numerical simulations of fictitious comets with a purely dynamical model and also with a more complete dynamical - physical model that includes besides nongravitational forces, sublimation and splitting mechanisms. We obtained better fits with models including physical effects, and in particular our best fits are for four splitting models with a relative weak dependence on $q$, and a mass loss in every splitting event that is smaller/greater for higher/lower frequencies respectively. The mean lifetime of JFCs with radii $R>1 \mathrm{~km}$ and $q<1.5 \mathrm{AU}$ is found to be of about $150-200$ revolutions $\left(\sim 10^{3} \mathrm{yr}\right)$. We find a total population of JFCs with radii $R>1 \mathrm{~km}$ within Jupiter's zone of $450 \pm 50$ and a mean lifetime of about $150-200$ revolutions $\left(\sim 10^{3} \mathrm{yr}\right)$ for those getting $q<1.5 \mathrm{AU}$. The population of JFCs + non-JFCs with radii $R>1 \mathrm{~km}$ in Jupiter-crossing orbits may be about $\sim 2,250 \pm 250$. Most of non-JFCs have perihelia close to Jupiter's orbit.
\end{abstract}

We also present maps of the densest zones of JFCs in the orbital element space.

Keywords. comets: general, methods: numerical.

\section{The observed population}

Tancredi et al. (2006) showed that the cumulative luminosity function (CLF) of JFCs can be well fitted by a straight line with a slope $0.54 \pm 0.05$ down to $H_{N}=16.7$, and then starts to flatten for fainter comets. Then the sample of observed JFCs seems to be complete down to $H_{N}=16.7$. The JFCs with $q<1.5 \mathrm{AU}$ and $H_{N}<16.7$ are 9 and there are 17 for $H_{N}<17.6$. This last population is incomplete. Fernández \& Morbidelli (2006) found that the CLF for the total magnitudes of JFCs follows a bimodal distribution, where the slope drops for fainter comets. They found a break in the distribution at a nuclear magnitude $H_{N} \sim 16.5-17.5$. Then we calculated, following those previous studies, the total number of JFCs with $q<1.5 \mathrm{AU}$ and $H_{N}<17.6$ at $N(17.6)=25 \pm 5$. From these 25 JFCs we have 17 observed JFCs and 8 whose orbital elements would be allocated in some way. Then we follow appropriate criteria for the incompleteness to assign the orbital elements of the remaining 8 JFCs. We will use the whole sample of 25 JFCs as our canonical data to compare with the numerical results obtained from our simulations. 


\section{The model}

Di Sisto \& Brunini (2007) performed numerical integrations of 1000 Scattered Disk (SD) objects (95 real +905 fictitious) with the aim of study their dynamical evolution as Centaurs. They follow the simulation under the gravitational action of the Sun and the four giant planets and stored in a file the orbital elements of the particles when they crossed Jupiter's orbit for the first time. Also they stored the orbital elements of the giant planets at every crossing. Here we considered as our initial particles the 218 objects that crossed Jupiter's orbit and performed series of numerical simulations but now adding the perturbations of the terrestrial planets, taking their mean anomalies at random. We made two groups of numerical runs:

First we numerically integrated the 218 initial objects and three more clones for each particle under the gravitational action of the Sun and the planets with EVORB (Fernández et al. 2002) with an integration step of $0.01 \mathrm{yr}$ for $10^{8} \mathrm{yr}$ or until collision with the Sun or a planet, or ejection.

Second we added in other three simulations the action of nongravitational forces, sublimation of ices and splitting effects. We used the 218 initial objects plus 218 clones, assigning an initial radius of $1 \mathrm{~km}, 5 \mathrm{~km}$ and $10 \mathrm{~km}$ to each particle in each integration. We followed the simulations until the comet reached a minimum radius of $100 \mathrm{~m}$, or collided with the Sun or a planet, or reached $q>6.3$ AU (transferred to the Centaur zone), or was ejected. We recorded the orbital elements of the test comets at perihelion.

The nongravitational forces were included as in Fernández et al. (2002). For the sublimation we fit a polynomial approximation to theoretical computations by Tancredi et al. (2006) of the mass loss per orbital revolution as a function of $q$, for $q<2.5 \mathrm{AU}$. We considered a standard value of the fraction of the active zone of the comet nucleus surface, $\nu=0.15$. Observations suggest that non-tidal splittings follow a certain trend to decrease for larger $q$. Then we try laws for the frequency of comet splittings of the kind $f=f_{0}\left(q / q_{0}\right)^{-\beta}$, where $\beta=0,0.5,1,1.5$ and $2, q_{0}=0.5$ AU and let $f_{0}$ as a free parameter. We propose a mass loss of the form $\Delta M=s M$ where $s=s(R)=\frac{s_{0}}{R / R_{0}}$. $s_{0}$ is the mass fraction of a comet with radius $R_{0}$ that is lost in a splitting event. The whole splitting model has then three free parameters: $\beta, s_{0}$ and $f_{0}$. We fix $R_{0}=10 \mathrm{~km}$ and $q_{0}=0.5 \mathrm{AU}$. We compared the orbital element distributions of our computed comet sample for each splitting model with the observed orbital element distribution of JFCs in order to define the free parameters.

\section{Results and conclusions}

In Fig. 1, we compare the orbital element distributions of JFCs obtained from the purely dynamical model, with observations. We can see that the test JFCs reach short perihelion distances and an inclination distribution broader than the observed one. Those features are artificial and a byproduct of the long lifetime of comets. For those JFCs we obtained mean lifetimes of 42,300 yr for $q<5.2 \mathrm{AU}, 16,300 \mathrm{yr}$ for $q<2.5 \mathrm{AU}$ and 7,600 in the zone $q<1.5 \mathrm{AU}$.

Then we included a complete model that takes into account nongravitational forces, sublimation and splitting. For the real size distribution of JFCs we adopted a bimodal cumulative size distribution for JFCs with $q<2.5$ AU given by

$$
\begin{array}{cc}
N_{1}(>R)=C_{1} R^{-1.3}, & 0.1 \mathrm{~km}<R \leqslant 1 \mathrm{~km} \\
N_{2}(>R)=C_{2} R^{-2.7}, & 1 \mathrm{~km} \leqslant R \leqslant 10 \mathrm{~km}
\end{array}
$$


We joined next the three dynamical - physical simulations picking out the JFCs with $q<2.5 \mathrm{AU}$ and assigning to each one of the outputs a weight in such a way that the whole sample follows the size distribution of JFCs. The weights $w_{i}$ so allocated represent the contribution of each instant comet, i.e. each comet revolution, to the whole sample of comet sizes. We shall call this the assembled sample. Comets of the assembled sample have radii between $0.1 \mathrm{~km}$ and $10 \mathrm{~km}$ and follow the observed size distribution of JFCs.

We run 52 splitting models with different sets of frequencies and mass losses, and compared the orbital element distribution to the observed sample. We have four best fits models where $\beta=1$ in two of them and 0.5 in the other two, $f_{0}$ goes from $1 / 6$ to 1 and $s_{0}$ is between 0.01 and 0.001 . We plot one of the best fit models as an example in Fig. 2. These models reflect a relative weak to moderate dependence on $q$, and a mass loss in every splitting event that is smaller/greater for higher/lower frequencies respectively. These frequencies of comet splittings are relatively high with respect to the observed major splittings. However since smaller splitting events may have passed undetected, they could increase the real frequency to make observations consistent with our results. Then, our results strongly suggest that splittings must be a frequent phenomenon in comets.

With regard to the end states, we find that comets with $1-\mathrm{km}$ initial radius mainly reach the minimum radius. This is due to the quick erosion that small comets suffer in our models reaching the minimum radius before they could evolve into other dynamical states. The most common final state of comets with $5-\mathrm{km}$ and $10-\mathrm{km}$ initial radius is the return to the Centaur region. We found that all the collisions occur with Jupiter.

In Fig. 3 we show maps that present the densest region, in the plane of orbital elements, where we would expect a greater number of JFCs. Those regions are the zones of $3<$ $a<4 \mathrm{AU}, 0.25<e<0.7,0^{\circ}<i<25^{\circ}$, and $2.7<T<3.05$. The observed JFCs tend to overlap the denser zones of our model.

With the complete dynamical - physical model we obtained mean lifetime of JFCs far smaller than the ones obtained through the dynamical simulation as shown above. For JFCs with radii $R>1 \mathrm{~km}$ we have a mean lifetime of $\sim 150-200$ revolutions $\left(\sim 10^{3} \mathrm{yr}\right)$ for $q<1.5 \mathrm{AU}(\sim 4.6$ times smaller), while for $q<2.5$ AU we have $\sim 300-450$ revolutions $\left(\sim 3 \times 10^{3} \mathrm{yr}\right)(\sim 5.7$ times smaller $)$. This is a product of the dominant role played by physical effects in the lifetimes of low- $q$ comets.

In Fig. 4 we represent the cumulative number of JFCs and of JFCs plus non-JFCs depending on $q$. We have found that the number of JFCs with radii $R>1 \mathrm{~km}$ inside
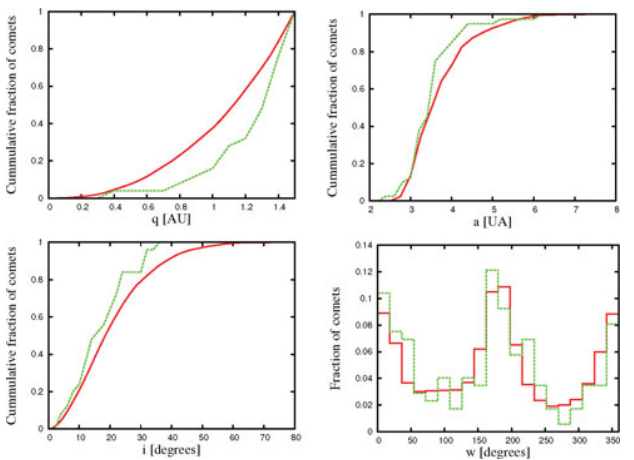

Figure 1. Orbital element distributions of JFCs from the dynamical model (solid line) and of JFCs with $q<1.5 \mathrm{AU}$ and $H_{N}<17.6$ (dashed line) (Di Sisto et al. (2009), Fig.5).
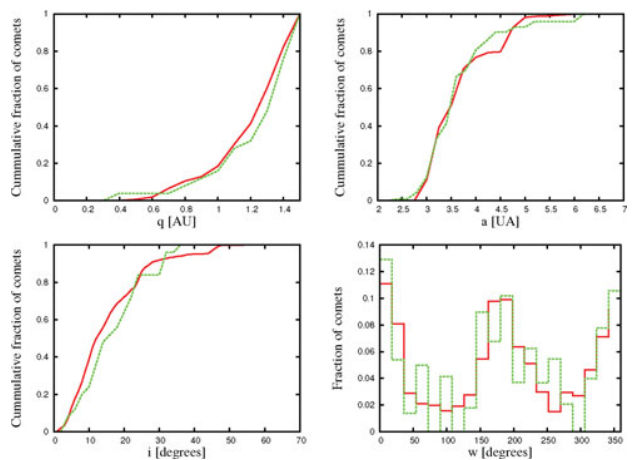

Figure 2. Orbital element distributions of JFCs from one of the best fit models (solid line) and of JFCs with $q<1.5 \mathrm{AU}, H_{N}<17.6$ (dashed line). 

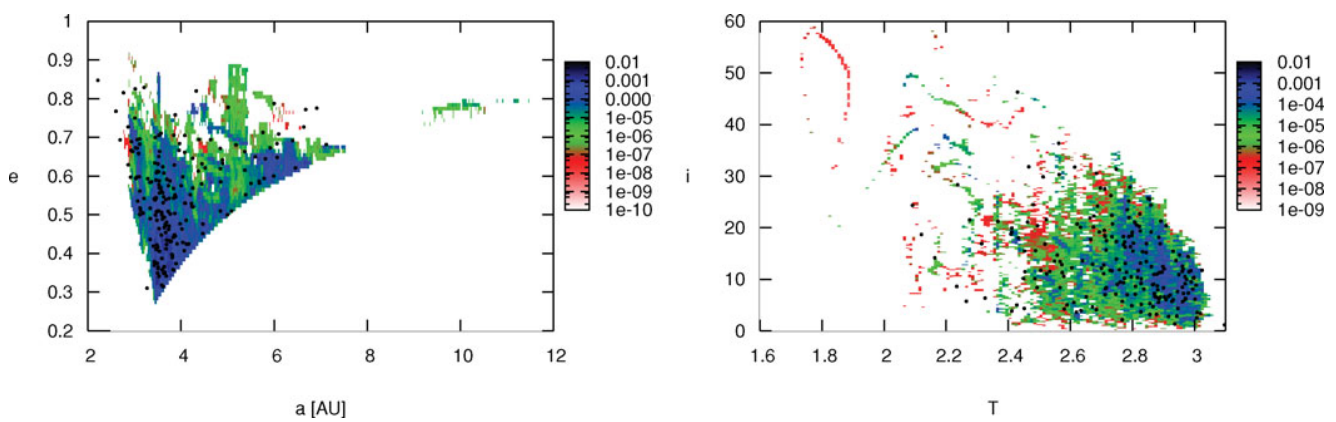

Figure 3. Time-weighted distribution of comets with $q<2.5$ AU obtained from from one of the best fit models in the orbital element space. The black circles are the observed JFCs.

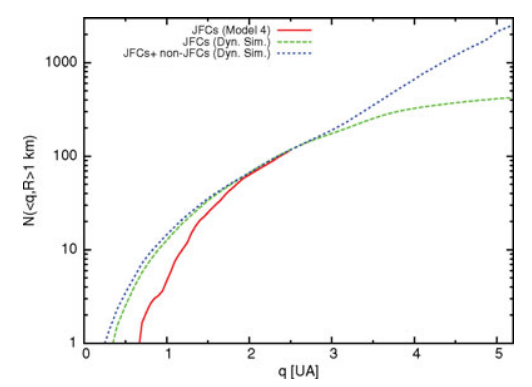

Figure 4. Cumulative number of JFCs and of JFCs+non-JFCs.(Di Sisto et al. (2009), Fig.8).

Jupiter's orbit is $450 \pm 50$. This is smaller by about one order of magnitude than some previous estimates (e.g. Fernández et al. 1999). For non-JFCs $(P>20$ yr) in Jupitercrossing orbits we obtained a number $\sim 4$ times greater that the number of JFCs $(1800 \pm$ 200). The perihelia of most of the non-JFCs is close to Jupiter's orbit.

The possibility that the observed JFCs with Tisserand parameters $T<2$ could come straight from the SD cannot be ruled out since we have some few JFCs that could reach a $T<2$ state through a peculiar long dynamical evolution. However this constitutes an unlikely event that requires long survival times as active comets.

In our simulations most comets that reach $q<2.5$ AU have orbital periods $P<20$ yr. Then we can conclude that Halley-type comets should have a different dynamical history, presumably requiring the passage by the Oort cloud prior to their plunging into the inner planetary region.

\section{References}

Di Sisto, R. P., Fernández, J. A., \& Brunini, A. 2009, Icarus, 203, 140

Di Sisto, R. P. \& Brunini, A. 2007, Icarus, 190, 224

Fernández, J. A., Gallardo, T., \& Brunini, A. 2002, Icarus, 159, 358

Fernández, J. A. \& Morbidelli, A. 2006, Icarus, 185, 211

Fernández, J. A., Tancredi, G., Rickman, H., \& Licandro, J. 1999, A\&A, 352, 327

Tancredi, G., Fernández, J. A., Rickman, H., \& Licandro, J. 2006, Icarus, 182, 527 